\begin{tabular}{|c|c|}
\hline Title & $\begin{array}{l}\text { A pplication of fluorescence resonance energy transfer (FRET) to investigation of light-induced conformational changes } \\
\text { of the phoborhodopsintransducer complex }\end{array}$ \\
\hline Author(s) & T aniguchi, Y ukinori; I kehara, Tatsuy a; Kamo, Naoki; W atanabe, Y asutaka; Y amasaki, Hiroshi; Toy oshima, Y oshinori \\
\hline Citation & $\begin{array}{l}\text { Photochemistry and Photobiology, 83(2), 311-316 } \\
\text { https://doi.org/10.1562/2006-06-15-RA-922 }\end{array}$ \\
\hline Issue Date & $2007-03$ \\
\hline Doc URL & http:/hdl.handle.net/2115/33764 \\
\hline Rights & $\begin{array}{l}\text { A uthor Posting. } \odot \text { The A uthors } 2007 \text { This is the author's version of the work. It is posted here for personal use, not for } \\
\text { redistribution. The definitive version was published in Photochemistry and Photobiology, V olume 83, Issue 2, Page } \\
\text { 311-316. http://dx.doi.org/10.1562/2006-06-15-RA-922 }\end{array}$ \\
\hline Type & article (author version) \\
\hline File Information & PA P83-2-311.pdf \\
\hline
\end{tabular}

Instructions for use 


\title{
Application of fluorescence resonance energy transfer (FRET) to investigation of light-induced conformational changes of the phoborhodopsin/transducer complex ${ }^{\dagger \ddagger}$
}

\author{
Yukinori Taniguchi $^{1 *}$, Tatsuya Ikehara ${ }^{1}$, Naoki Kamo ${ }^{2}$, Yasutaka Watanabe ${ }^{3}$, \\ Hiroshi Yamasaki ${ }^{1}$ and Yoshinori Toyoshima ${ }^{1}$
}
${ }^{1}$ Nano-biotechnology Research Center, and Department of Bioscience, School of Science \& Technology, Kwansei Gakuin University, Sanda, Hyogo, Japan.
${ }^{2}$ Graduate School of Pharmaceutical Sciences, Hokkaido University, Sapporo, Hokkaido, Japan.

${ }^{3}$ Department of Physics, School of Science \& Technology, Kwansei Gakuin University, Sanda, Hyogo, Japan.
*corresponding author: Yukinori Taniguchi
E-mail: guchi@,ksc.kwansei.ac.jp
${ }^{\dagger}$ Presented in part at the $12^{\text {th }}$ International Conference on Retinal Proteins, Awaji Island, Hyogo, Japan, 4 - 8 June, 2006.

\footnotetext{
$\$$ Published in the journal website (www.ASPjournal.com) on $\mathrm{xx}-\mathrm{xxx}-\mathrm{xxx}$.
}

* To whom correspondence should be addressed. 


\section{ABSTRACT}

The photoreceptor phoborhodopsin ( $p$ pR; also called sensory rhodopsin II) forms a complex with its cognate the Halobacterial transducer II ( $p$ HtrII) in the membrane, through which changes in the environmental light conditions are transmitted to the cytoplasm in Natronomonas pharaonis to evoke negative phototaxis. We have applied a fluorescence resonance energy transfer (FRET)based method for investigation of the light-induced conformational changes of the $p \mathrm{pR} / p \mathrm{HtrII}$ complex. Several far-red dyes were examined as possible fluorescence donors or acceptors because of the absence of the spectral overlap of these dyes with all the photointermediates of $p \mathrm{pR}$. The flash-induced changes of distances between the donor and an acceptor linked to cysteine residues which were genetically introduced at given positions in $p \mathrm{HtrII}(1-159)$ and $p \mathrm{pR}$ were determined from FRET efficiency changes. The dye-labeled complex was studied as solubilized in $0.1 \%$ n-dodecyl- $\beta$-D-maltoside (DDM). The FRET-derived changes in distances from V78 and A79 in $p$ HtrII to V185 in $p p R$ were consistent with the crystal structure data [Moukhametzianov, R. et al. (2006) Nature, 440, 787-792]. The distance from D102 in pHtrII linker region to V185 in $p \mathrm{pR}$ increased by $0.33 \AA$ upon the flash excitation. These changes arose within $70 \mathrm{~ms}$ (the dead time of instrument) and decayed with a rate of $1.1 \pm 0.2 \mathrm{~s}$. Thus, sub angstrom-scale distance changes in the $p \mathrm{pR} / p$ HtrII complex were detected with this FRET-based method using far-red fluorescent dyes; this method should be a valuable tool to investigate conformation changes in the transducer, in particular its dynamics. 


\section{INTRODUCTION}

Phoborhodopsin ( $p$ pR; also called sensory rhodopsin II) is a photoreceptor for negative phototaxis in Natronomonas pharaonis. $\quad p \mathrm{pR}$ and its cognate transducer (Halobacterium transducer II; $p \mathrm{HtrII}$ ) form a 2:2 complex in which $p \mathrm{HtrII}$ dimer is sandwiched between two $p \mathrm{pR}$ molecules in the plasma membrane $(1,2)$. The $p \mathrm{pR} / p$ HtrII complex mediates the light signal to cytoplasmic signal transduction pathway in the earliest step of phototaxis. The $p$ HtrII is a homolog of the chemotaxis receptor (methyl-accepting chemotaxis protein: MCP) of E. coli and is thought to be complexed with a histidine kinase CheA and adaptor protein $\mathrm{CheW}$ in the cytoplasm. The structures of the transmembrane region of the complex in its initial state (2) and for the $\mathrm{K}$ and $\mathrm{M}$ states (3) were determined by X-ray crystallography with atomic resolution. The cytoplasmic region of $p H$ trII has been thought to be a four-helical-bundle coiled-coil structure similar to that of the bacterial chemoreceptor (4). However, the structure of the $p H$ trII linker region, which connects the transmembrane region and the cytoplasmic region, is not as well understood although it has been investigated by $\operatorname{NMR}(5,6), \operatorname{EPR}(7,8)$ and tryptophan FRET $(9)$.

Light-induced conformational changes in $p H$ trII of the complex have been suggested to occur as a rotation and tilt of transmembrane helix 2 (TM2) $(1,3,10)$. It was also suggested that $\mathrm{S} 154$ in the E-F loop of $p \mathrm{pR}$ is brought close to membrane proximal region (91-95) of $p$ HtrII in the photoactivated complex (9). However, no one has shown light-induced conformational changes of the linker region and the cytoplasmic region of $p \mathrm{HtrII}$.

In this study, we have applied a fluorescence resonance energy transfer (FRET)-based method to investigate the light-induced site-specific distance changes in the complex. FRET is a 
distance-dependent energy transfer from an excited fluorescent dye to an acceptor in the ground state; this provides a convenient spectroscopic method to study changes in the structure of the protein complex (11-15). However, because of the presence of spectral overlap of the dyes used with the absorption spectrum of the intermediates of $p \mathrm{pR}$ per se, quantitative analysis of FRET experiments is difficult for the $p \mathrm{pR} / p \mathrm{HtrII}$ complex. To overcome this problem, we examined several recently-developed far-red fluorescent dyes, Cy5 and Alexa660 as fluorescent donors and Cy7Q, HiLyte Fluor 750 (HF750) and DY750 as acceptors. The fluorescence and absorption spectra of these dyes hardly overlap with the absorption spectra of the $p \mathrm{pR}$ intermediates as shown in Fig. 1. The donor and acceptor dyes were linked to cysteine residues genetically introduced at given positions in $p \mathrm{HtrII}(1-159)$ and $p \mathrm{pR}$, respectively. The changes in the distances between the donor and acceptor for various pairs upon flash excitation of $p \mathrm{pR}$ were measured in the presence of $0.1 \% \mathrm{n}$-dodecyl- $\beta$-D-maltoside (DDM).

\section{MATERIALS AND METHODS}

Reagents. Thiol reactive dyes, Alexa $660 \mathrm{C}_{2}$-maleimide, HiLyte Fluor 750- $\mathrm{C}_{2}$-maleimide and DY750-maleimide were purchased from Invitrogen Corp. (Carisbad, CA, USA), AnaSpec, Inc, (San Jose, CA, USA) and Dyomics GmbH. (Jena, Germany), respectively. Cy5-maleimide and Cy7Q-maleimide were from GE Healthcare Bio-Sciences Corp. (Piscataway, NJ, USA). The detergents, n-dodecyl- $\beta$-D-maltoside (DDM) and n-octyl- $\beta$-glocoside (OG) were from Dojindo laboratories (Kumamoto, Japan). All-trans retinal, isopropyl-1-thio- $\beta$-D-galactopyranoside (IPTG) and lysozyme were from Wako Pure Chemical Industries, Ltd. (Osaka, Japan). Nile blue sulfphate was from Waldeck GmbH \& Co. KG (Münster, Germany). 
Construction of plasmids. Expression plasmids of $p \mathrm{pR}$ and $p \mathrm{HtrII}(1-159)$ possessing $6 \mathrm{x}$ histidinetag at the C-terminus were constructed previously (16). Expression plasmids for $p \mathrm{pR}$ and $p \mathrm{HtrII}(1-159)$ with single cysteine substitution at various positions were prepared by PCR using the QuikChange method. Introduction of the single mutation was confirmed by sequence analysis using an automated sequencer, PRISM 3100-Avant and BigDye Terminator v3.1 Cycle Sequencing Kit (Applied Biosystems).

Protein expression and purification. Wild-type and mutant proteins of $p \mathrm{pR}$ and $p \mathrm{HtrII}(1-159)$ were expressed in E. coli BL21(DE3) cultured in 2xYT medium by IPTG (1mM) induction (17). Concomitantly with the addition of IPTG, all-trans retinal was added (final concentration was $10 \mu \mathrm{M})$. Harvested cells were resuspended in buffer A (50mM Tris $\mathrm{pH} 8.0,5 \mathrm{mM} \mathrm{MgCl}$, $0.5 \mathrm{mg} / \mathrm{ml}$ lysozyme) and lysed by sonication. The lysate was centrifuged at $100,000 \mathrm{~g}$ for $1.5 \mathrm{~h}$, and then the membrane fraction collected and solubilized with 1\% OG in buffer S (50mM MES pH $6.5,300 \mathrm{mM} \mathrm{NaCl}, 5 \mathrm{mM}$ imidazole) at $4^{\circ} \mathrm{C}$ overnight. After centrifugation of the solution containing solubilized proteins at $100,000 \mathrm{~g}$ for $1.5 \mathrm{~h}$, the supernatant was collected and incubated with Ni-NTA agarose (Qiagen) for $1 \mathrm{~h}$ at $4^{\circ} \mathrm{C}$. The Ni-NTA resin was washed with buffer W (50 $\mathrm{mM}$ MES pH 6.5, 300mM NaCl, 25 or 100mM imidazole, 0.1\% DDM), and His-tagged protein eluted with buffer E (50mM Tris pH 7.0, 300mM NaCl, 300mM imidazole, 0.1\% DDM) (17). In the case of $p \mathrm{pR}$, further purification with ion-chromatography was performed. The effluent from the Ni-NTA resin was incubated with DEAE-sephacel (GE Healthcare) in buffer B (50mM HEPES $\mathrm{pH} 7.0,0.1 \%$ DDM) for $1 \mathrm{~h}$ at room temperature and $p \mathrm{pR}$ was eluted with buffer B containing $50-100 \mathrm{mM} \mathrm{NaCl}$. The purity of the $p \mathrm{pR}$ and $p \mathrm{HtrII}$ was checked by SDS-PAGE, and 
further the ratio of absorbance at $500 \mathrm{~nm}$ to that at $280 \mathrm{~nm}$ in the case of $p \mathrm{pR}$. SDS-PAGE of the purified $p \mathrm{pR}$ and $p \mathrm{HtrII}$ showed single bands corresponding to 25 and $17-\mathrm{kDa}$, respectively.

Protein labeling. Each of purified proteins was incubated with 10-fold excess of Tris[2carboxyethyl]phosphine (TCEP) in 100mM HEPES pH 7.6 for $2 \mathrm{~h}$ at room temperature to reduce disulfide bonds. Then, a 10-fold excess of a thiol-reactive dye was added and the mixture incubated for $2 \mathrm{~h}$ at room temperature. Dye-labeled proteins were separated from free dye by gelfiltration with Bio-Gel P-6 (BioRad) in the cases of Alexa660 and Cy5. In the case of Cy7Q, HF750 and DY750, the separation was performed by affinity chromatography with the Ni-NTA column. The concentrations of $p \mathrm{pR}$ and dyes were determined by measuring their absorbances and that of $p$ HtrII was done by using the Micro BCA Protein Assay Kit (Pierce) and labeling efficiency was calculated from their values for each sample preparation. The labeling efficiencies $(\gamma)$ of acceptor were $0.68 \sim 0.84$

Measurement of FRET efficiency. The relation of FRET efficiency $(E)$ to the distance between donor and acceptor $(R)$ is given by the equation,

$$
E=\frac{R_{0}^{6}}{R_{0}^{6}+R^{6}},
$$

where $R_{0}$ is the Förster distance. The values of the Förster distances were calculated from equation 2, which depends on the donor and acceptor pairs used (18),

$$
R_{0}^{6}=\frac{9000(\ln 10) \kappa^{2} \psi_{d}}{128 \pi^{5} n^{4} N_{A}} \int \varepsilon_{a}(\lambda) F_{d}(\lambda) \lambda^{4} d \lambda,
$$

where $\kappa^{2}$ is a factor for the relative orientation of the transition moments of dyes, $\psi_{d}$ is 
fluorescence quantum yield of donor, $n$ is the refractive index, $N_{A}$ is Avogadro's number, $F_{d}(\lambda)$ is the normalized fluorescence of donor, $\varepsilon_{a}(\lambda)$ is the molar extinction coefficient of acceptor and $\lambda$ is the wavelength. The integral was determined for each pair by measuring the fluorescence spectrum of donor and the absorbance spectrum of acceptor with a fluorescence spectrophotometer (Hitachi, F-4500) and a UV/VIS spectrophotometer (Hitachi, U-3500), respectively. The fluorescence quantum yield of a donor linked to a given cysteine residue was determined using Nile blue as a standard. Assuming isotropic orientation of donor and acceptor dyes, $\kappa^{2}$ was taken to be $2 / 3$.

To obtain the FRET efficiency in which the effect of the absorption of the donor fluorescence by the acceptor was eliminated, the fluorescence intensity in a solution of the $p \mathrm{pR}$ acceptor $/ p$ HtrII-donor complex $\left(I_{c o n j}\right)$ was measured concomitantly with that in a solution containing a non-labeled $p \mathrm{pR} / p \mathrm{HtrII}$-donor complex and the free acceptor at their corresponding concentrations $\left(I_{\text {cont }}\right)$. In the control, FRET was expected not to occur because the average distance between the donor and the bulk acceptor was sufficiently long compared to the Förster distance. Hence, FRET efficiency was determined experimentally based on the equation,

$$
E=\frac{1}{\gamma} \frac{I_{c o n t}-I_{c o n j}}{I_{c o n t}},
$$

where $\gamma$ is labeling efficiency of acceptor, which was determined for each sample preparation independently. $I_{c o n j}$ and $I_{c o n t}$ were measured at the wavelength giving the maximum of the donor fluorescence (Cy5: 670nm, Alexa660: 690nm) at room temperature. The samples were incubated in Buffer M (50mM HEPES pH 7.0, 400mM NaCl, 0.1\% DDM) for at least $3 \mathrm{~h}$ to form a complex prior to the fluorescence measurements. 
Time-resolved FRET measurement. A fluorescence spectrometer associated with a flash apparatus was assembled to measure transient change of fluorescent intensity upon photoexcitation of $p \mathrm{pR}$. The fluorescence donor was excited at $633 \mathrm{~nm}$ by using a continuous He-Ne laser (Neoarc, HN550R). Fluorescence was passed through a long pass filter (Fujifilm, SC64) and a monochrometor (Jobin Yvon, HR320), and detected by a photomultiplier tube (Hamamatsu photonics, R1463). After passing an electronic low-pass filter (cutoff frequency: 100Hz) for reduction of highfrequency noise, the signal was digitized by a digital synchroscope (Tektronix, TDS2002). Actinic light was provided by a Xe flash lamp (Sunpak, Auto544) in combination with a 500nm band-pass filter (Fujifilm, BPB50). To avoid the presence of labeled $p$ HtrII which is not associated with labeled $p \mathrm{pR}, 8 \mu \mathrm{M}$ of labeled $p \mathrm{pR}$ and $4 \mu \mathrm{M}$ of labeled $p \mathrm{HtrII}$ was incubated in Buffer $M$ at least $3 \mathrm{~h}$ at room temperature, and submitted to the fluorescent measurement. All measurements were carried out at $20^{\circ} \mathrm{C}$; data from the average of 160 flashs was used.

The apparent FRET efficiency at time $t\left(E_{\text {app }}(t)\right)$ was calculated by the equation,

$$
E_{\text {app }}(t)=\frac{1}{\gamma} \frac{I_{c o n t}(t)-I_{c o n j}(t)}{I_{c o n t}(t)}
$$

and the distance change $(\Delta R(t))$ was obtained by using the formula,

$$
\frac{\Delta R(t)}{R}=-\frac{1}{\psi} \frac{\Delta E_{a p p}(t)}{6 E(1-E)},
$$

derived from eq. 1 , assuming $\Delta R(t) \quad R$. Here, $E$ is the FRET efficiency at the initial state, $\Delta E_{\text {app }}(t)$ $\left(=E_{\text {app }}(t)-E\right)$ is apparent FRET efficiency change at time $t$ and $\psi$ is the excitation efficiency of $p \mathrm{pR}$ upon the flash, which is dependent on the arrangement of the apparatus. The value of $\psi$ was determined as shown in Table 1 from the relative reduction of the $p \mathrm{pR}$ initial state upon the flash, obtained by measuring the absorbance change at 500nm. 


\section{RESULTS}

\section{FRET-derived distances between a donor and acceptor linked to given residues in the $p \mathrm{pR} / \mathrm{pHtrII}$ complex}

The fluorescence spectrum of a solution containing $8 \mu \mathrm{M}$ of acceptor-labeled $p \mathrm{pR}$ and $4 \mu \mathrm{M}$ of donor-labeled $p H \operatorname{HrII}$ (conjugate) in the presence of $0.1 \%$ DDM was compared with that of a solution containing $8 \mu \mathrm{M}$ of non-labeled $p \mathrm{pR}, 4 \mu \mathrm{M}$ of donor-labeled $p \mathrm{HtrII}$ and $8 \gamma \mu \mathrm{M}$ of free acceptor (control) for the donor and acceptor pair of Cy5/Cy7Q and Alexa660/Cy7Q in Fig. 2. In the conjugate sample of $p \mathrm{pR}-\mathrm{V} 185 \mathrm{C}-\mathrm{Cy} 7 \mathrm{Q} / \mathrm{pHtrII}-\mathrm{A} 79 \mathrm{C}-\mathrm{Cy} 5$, the donor fluorescence (670nm) became lower than that of the control sample concomitant with the increase of the acceptor fluorescence (780nm, Fig. 2a). Similar behavior was observed in the other combinations of donors and acceptors, such as Alexa660/Cy7Q (Fig. 2b), Cy5/HF750 and Cy5/DY750 (data not shown). These results indicated that $p \mathrm{pR}$ and $p \mathrm{HtrII}$ form a complex in the presence of $0.1 \%$ DDM and a part of energy absorbed by the donor was transferred to the acceptor through FRET process. The FRET-derived distances between the donor and acceptor in $p \mathrm{pR}-\mathrm{V} 185 \mathrm{C}$ -

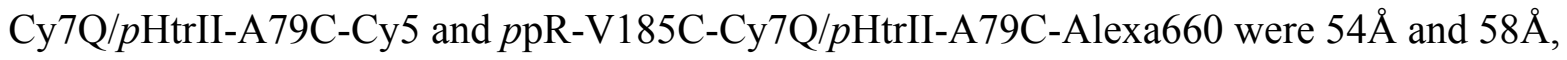
respectively. This difference may be due to the difference in size and/or averaged orientation of these donor dyes. When the concentrations of $p \mathrm{pR}-\mathrm{V} 185 \mathrm{C}-\mathrm{Cy} 7 \mathrm{Q}$ in the solution was increased to $12 \mu \mathrm{M}$ at the fixed concentration of $p$ HtrII-A79C-Cy5 $(4 \mu \mathrm{M})$, FRET efficiency increased and saturated at $4 \mu \mathrm{M}$ giving $\mathrm{E}=0.73$ as shown in Fig. 3. FRET efficiency of 0.73 corresponds to $54 \AA$ for the distance $(\mathrm{R})$ between the donor and acceptor on the basis of eq. $1\left(\mathrm{R}_{0}=64 \AA\right)$. In the case of 
$p$ pR-V58C-Cy7Q/pHtrII-A79C-Cy5, the saturation level of FRET efficiency is 0.51 which corresponds to a distance of $64 \AA$. The difference between them is $10 \AA$ which is consistent with the difference in the $\mathrm{C}_{\alpha}$ distances between $p \mathrm{pR}-\mathrm{V} 185 / p \mathrm{HtrII}-\mathrm{A} 79$ pair and $p \mathrm{pR}-\mathrm{V} 58 / p \mathrm{HtrII}-\mathrm{A} 79$ pair (7Å) determined by X ray crystallography (2). This suggests that the FRET-derived distance between a donor and an acceptor linked to the given amino acids reflects the distance between the $\mathrm{C}_{\alpha} \mathrm{S}$ of the corresponding residues.

\section{FRET-derived distance change in the $p p R / p H$ trII complex upon flash excitation of $p p R$}

Flash-induced change of fluorescence intensity in the conjugate and control samples of $p \mathrm{pR}-$ V185C-Cy7Q/pHtrII-D102C-Alexa660 and $p$ pR-V185C-Cy7Q/pHtrII-D102C-Cy5 were examined and the results are shown in Fig. 4. Here, $p$ pR-V185 is located in the extracellular F-G loop and $p$ HtrII-D102 is in the putative loop of linker region. The fluorescence intensity of the conjugate sample of $p \mathrm{pR}-\mathrm{V} 185 \mathrm{C}-\mathrm{Cy} 7 \mathrm{Q} / \mathrm{pHtrII-D102C-Alexa660} \mathrm{increased} \mathrm{within} \mathrm{70ms} \mathrm{(the} \mathrm{dead}$ time of instrument) and returned to the initial state level at a rate of approximately $1.1 \mathrm{~s}$. In the control sample, no change was observed. When these samples were illuminated with 600nm-flash light, which is not absorbed by $p \mathrm{pR}$, no change was observed (data not shown). These facts indicate that the change of fluorescence intensity takes place in response to the photoactivation of $p p$ R. The apparent FRET efficiency at time $t\left(E_{\text {app }}(t)\right)$ calculated on the basis of eq. 4 is shown in Fig. 4c. When Cy5 was used as a donor instead of Alexa660, fluorescence intensity of the control sample decreased upon flash excitation and the change of fluorescence intensity disappeared in the conjugate sample as shown in Fig. $4 \mathrm{~b}$. However, $E_{\text {app }}(t)$ calculated on the basis of eq. 4 was almost same as the case of the donor and acceptor pair of Alexa660/Cy7Q as shown in Fig. 4c and 
d. The flash-induced transient decrease of Cy5 fluorescence in the control sample (non-labeled $p \mathrm{pR}-\mathrm{V} 185 \mathrm{C} / p \mathrm{HtrII}-\mathrm{D} 102 \mathrm{C}-\mathrm{Cy} 5 /$ free Cy7Q) is not due to the absorption of the emitted Cy5 fluorescence by the light-induced O-intermediate of $p \mathrm{pR}$, because it was not observed in free Cy5/free $p$ pR solution. The magnitude of the fluorescence decrease varied depending on the site of Cy5-labeling (data not shown), suggesting that the transient fluorescence decrease in the control sample arises from FRET to the $\mathrm{O}$-intermediate of $p \mathrm{pR}$. The values of $\mathrm{R}_{0}$ calculated using the absorption spectrum of the O-intermediate have previously been reported (19) as $43 \AA$ and $32 \AA$ for Cy5/ppR-O and Alexa660/ppR-O pairs, respectively. This difference in the value of $\mathrm{R}_{0}$ might be responsible for the difference in the efficiency of FRET to the O-intermediate of $p \mathrm{pR}$ from the donor dye. That is, FRET from donor dye in the complex to the O-intermediate of $p \mathrm{pR}$ is stronger in the case of Cy5 than Alexa660. Alternatively, the flash-induced fluorescence depletion in the control sample (non-labeled $p \mathrm{pR}-\mathrm{V} 185 \mathrm{C} / p \mathrm{HtrII}-\mathrm{D} 102 \mathrm{C}-\mathrm{Cy} 5 /$ free Cy7Q) might be due to a flashinduced micro environmental change, such as a change in the hydrophobicity around Cy5.

The light-induced relative movement of V78 or A79 in transmembrane helix 2 of $p H \operatorname{trII}$ with respect to V185 in $p$ pR was also examined using the donor and acceptor pair Alexa660/Cy7Q. Similar to the case of D102 in $p H t r I I$, FRET efficiency in $p p R-V 185 C$ Cy7Q/pHtrII-V78C-Alexa660 and ppR-V185C-Cy7Q/pHtrII-A79C-Alexa660 decreased within $70 \mathrm{~ms}$ and recovered to the initial state level at a rate of approximately $1.1 \mathrm{~s}$ upon flash excitation, although the absolute value of the change was different from each other, which are summarized in Table. 1. These results suggest that a light-induced conformational change occurs before or at the time of formation of the M state and returned to the initial state concomitantly with the decay of the $\mathrm{M}$ state or the $\mathrm{O}$ state of $p \mathrm{pR}$. 


\section{DISCUSSION}

We have developed a FRET-based method to investigate site-specific distance changes and their dynamics in the $p \mathrm{pR} / p \mathrm{HtrII}$ complex formed in the presence of $0.1 \%$ DDM. By using far-red fluorescent dyes, FRET efficiency was determined without disturbance by possible absorption of any of the $p \mathrm{pR}$ intermediates during photocycle. To eliminate the effect of absorption of the donor fluorescence by the acceptor, the intensity of donor fluorescence in the solution containing non-labeled $p \mathrm{pR} /$ donor-labeled $p \mathrm{HtrII}$ complex and free acceptor was measured as control. In the control solution, FRET was expected not to occur because the average distance between the donor and the bulk acceptor was sufficiently long compared to the Förster distance. The estimation of distance and its change from FRET efficiency was performed assuming the 1:1 complex of $p \mathrm{pR}$ and $p \mathrm{HtrII}$, because previous studies have shown that stoichiometry of the complex in the detergent solution was 1:1 $(8,20,21)$, instead of the 2:2 complex formed in lipid membranes $(1,2)$. For simplifying analysis, the distance change upon flash excitation was determined by using the donor and acceptor pair of Alexa660/Cy7Q instead of Cy5/Cy7Q, although the flash-induced change of FRET efficiency was almost identical in both the donor and acceptor pairs.

The light-induced distance changes calculated from FRET efficiency change determined for V78 and A79 in $p \mathrm{HtrII}$ against V185 in $p$ R are compared with the distance changes of the corresponding pairs calculated from the recently reported crystal structure data (3) in Table. 1. They are consistent with each other, suggesting the validity of the present FRET-based method for detection of sub-angstrom scale light-induced conformational change of the $p \mathrm{pR} / p \mathrm{HtrII}$ complex. It should be noted, however, that the FRET-derived distance change may not exactly reflect the amino acid distance change in crystal structure because of environmental difference of the 
complex in $0.1 \%$ DDM solution and in crystal, and the bulkiness and mobility of the dyes. The present FRET-based method should be a useful tool to investigate conformational changes and their dynamics for the linker region and the cytoplasmic region of $p \mathrm{HtrII}$, which were not detected in $\mathrm{X}$ ray crystallography.

Acknowledgments. This work has been supported in part by Kwansei Gakuin University for Special Individual Research. 


\section{REFERENCES}

1. Wegener, A.-A., J. P. Klare, M. Engelhard and H.-J. Steinhoff (2001) Structural insights into the early steps of receptor-transducer signal transfer in archaeal phototaxis. EMBO J. 20, $5312-5319$.

2. Gordeliy, V. I., J. Labahn, R. Moukhametzianov, R. Efremov, J. Granzin, R. Schlesinger, G. Büldt, T. Savopol, A. J. Scheidig, J. P. Klare and M. Engelhard (2002) Molecular basis of transmembrane signaling by sensory rhodopsin II-transducer complex. Nature. 419, 484-487.

3. Moukhametzianov, R., J. P. Klare, R. Efremov, C. Baeken, A Göppner, J. Labahn, M. Engelhard, G. Büldt and V. I. Gordeliy (2006) Development of the signal in sensory rhodopsin and its cognate transducer. Nature. 440, 115-119.

4. Kim, K. K., H. Yokota and S.-H. Kim (1999) Four-helical-bundle structure of the cytoplasmic domain of a serine chemotaxis receptor. Nature. 400, 787-792.

5. Yamaguchi, S., K. Shimono, Y. Sudo, S. Tuzi, A. Naito, N. Kamo and H. Saitô (2004) Conformation and dynamics of the $\left[3-{ }^{13} \mathrm{C}\right] \mathrm{Ala},\left[1-{ }^{13} \mathrm{C}\right]$ Val-labeled truncated pharaonis transducer, $p \mathrm{HtrII}(1-159)$, as revealed by site-directed ${ }^{13} \mathrm{C}$ solid-state NMR: changes due to association with phoborhodopsin (sensory rhodopsin II). Biophys. J. 86, 3131-3140.

6. Sudo, Y., H. Okuda, M. Yamabi, Y. Fukuzaki, M. Mishima, N. Kamo and C. Kojima (2005) Linker region of a halobacterial transducer protein interacts directly with its sensor retinal protein. Biochemistry. 44, 6144-6152.

7. Bordignon, E., J. P. Klare, M. Doebber, A. A. Wegener, S. Martell, M. Engelhard and H.-J. Steinhoff (2005) Structural analysis of a HAMP domain. J. Biol. Chem. 280, 38767-38775.

8. Klare, J. P., E. Bordignon, M. Doebber, J. Fitter, J. Kriegsmann, I. Chizhov, H.-J. Steinhoff 
and M. Engelhard (2006) Effects of solubilization on the structure and function of the sensory rhodopsin II/transducer complex. J. Mol. Biol. 356, 1207-1221.

9. Yang, C.-S., O. Sineshchekov, E. N. Spudich and J. L. Spudich (2004) The cytoplasmic membrane-proximal domain of the HtrII transducer interacts with the E-F loop of photoactivated Natoronomonas pharaonis sensory rhodopsin II. J. Biol. Chem. 279, 4297042976.

10. Klare, J. P., V. I. Gordeliy, J. Labahn, G. Büldt, H.-J. Steinhoff and M. Engelhard (2004) The archaeal sensory rhodopsin II/transducer complex: a model for transmembrane signal transfer. FEBS Lett. 564, 219-224.

11. Medintz, I. L., J. H. Konnert, A. R. Clapp, I. Stanish, M. E. Twigg, H. Mattoussi, J. M. Mauro and J. R. Deschamps. (2004) A fluorescence resonance energy transfer-derived structure of a quantum dot-protein bioconjugate nanoassembly. Proc. Natl. Acad. Sci. 101, 9612-9617.

12. Ramachandran, R., R. K. Tweten and A. E. Johnson (2005) The domains of a cholesteroldependent cytolysin undergo a major FRET-detected rearrangement during pore formation. Proc. Natl. Acad. Sci. 102, 7139-7144.

13. Hickerson, R., Z. K. Majumdar, A. Baucom, R. M. Clegg and H. F. Noller (2005) Measurement of internal movements within the $30 \mathrm{~S}$ ribosomal subunit using Förster resonance energy transfer. J. Mol. Biol. 354, 459-472.

14. Posson, D. J., P. Ge, C. Miller, F. Bezanilla and P. R. Selvin (2005) Small vertical movement of a $\mathrm{K}^{+}$channel voltage sensor measured with luminescence energy transfer. Nature. 436, 848851.

15. Chanda, B., O. K. Asamoah, R. Blunck, B. Roux and F. Bezanilla (2005) Gating charge displacement in voltage-gated ion channels involves limited transmembrane movement. 
Nature. 436, 852-856.

16. Kandori, H., K. Shimono, Y. Sudo, M. Iwamoto, Y. Shichida and N. Kamo (2001) Structural changes of pharaonis phoborhodopsin upon photoisomerization of the retinal chromophore: infrared spectral comparison with bacteriorhodopsin. Biochemistry. 40, 9238-9246.

17. Shimono, K., M. Iwamoto, M. Sumi and N. Kamo (1997) Functional expression of pharaonis phoborhodopsin in Eschericha coil. FEBS Lett. 420, 54-56.

18. Lakowicz, J. R. (1983) Principles of Fluorescence Spectroscopy. Plenum Press, New York.

19. Miyazaki, M., J. Hirayama, M. Hayakawa and N. Kamo (1992) Flash photolysis study on pharaonis phoborhodopsin from a haloalkaliphilic bacterium (Natronobacterium pharaonis). Biochim. Biophys. Acta. 1140, 22-29.

20. Sudo, Y., M. Iwamoto, K. Shimono and N. Kamo (2001) Pharaonis phoborhodopsin binds to its cognate truncated transducer even in the presence of a detergent with a 1:1 stoichiometry. Photochem. Photobiol. 74, 489-494.

21. Hippler-Mreyen, S., J. P. Klare, A. A. Wegener, R. Seidel, C. Herrmann, G. Schmies, G. Nagal, E. Bamberg and M. Engelhard (2003) Probing the sensory rhodopsin II binding domain of its cognate transducer by calorimetry and electrophysiology. J. Mol. Biol. 330, 1203-1213. 


\section{FIGURE CAPTIONS}

Figure 1. (a) Fluorescence spectra of donors (Cy5 and Alexa660) and absorption spectra of acceptors (Cy7Q, HF750 and DY750) examined in this study. (b), (c) and (d) Molecular structures of Cy5-maleimide, Cy7Q-maleimide and DY750-maleimide, respectively. The structures of Alexa660 and HF750 cannot be shown because they are proprietary information.

Figure 2. Fluorescence spectra of the conjugate and control samples (see text). (a) $p p R-$ V185C-Cy7Q/pHtrII-A79C-Cy5. (b) $p$ pR-V185C-Cy7Q/pHtrII-A79C-Alexa660. The concentrations of $p \mathrm{pR}$ and $p \mathrm{HtrII}$ were $8 \mu \mathrm{M}$ and $4 \mu \mathrm{M}$, respectively.

Figure 3. FRET efficiency at various concentrations of $p \mathrm{pR}$ under the condition of a fixed $p \mathrm{HtrII}$ concentration of $4 \mu \mathrm{M}$. (open diamonds) $p \mathrm{pR}-\mathrm{V} 185 \mathrm{C}-\mathrm{Cy} 7 \mathrm{Q} / p \mathrm{HtrII}-\mathrm{A} 79 \mathrm{C}-\mathrm{Cy} 5$. (filled diamonds) $p$ pR-V58C-Cy7Q/pHtrII-A79C-Cy5.

Figure 4. The changes of intensity of donor fluorescence ( $a$ and $b$ ) and FRET efficiency (c and d) upon flash excitation of $p \mathrm{pR}$. (a and c) $p \mathrm{pR}-\mathrm{V} 185 \mathrm{C}-\mathrm{Cy} 7 \mathrm{Q} / p \mathrm{HtrII}-\mathrm{D} 102 \mathrm{C}-\mathrm{Alexa660.}$ (b and d) $p \mathrm{pR}-\mathrm{V} 185 \mathrm{C}-\mathrm{Cy} 7 \mathrm{Q} / p \mathrm{HtrII}-\mathrm{D} 102 \mathrm{C}-\mathrm{Cy} 5$. Fluorescence intensity was monitored at 690nm for Alexa660/Cy7Q pair or 670nm for Cy5/Cy7Q pair. Each trace was an average of 160 flash data recorded at $20^{\circ} \mathrm{C}$. The very sharp increase and decrease within $70 \mathrm{~ms}$ in (a) and (b) were an instrumental artifact. The concentrations of $p \mathrm{pR}$ and $p \mathrm{HtrII}$ were $8 \mu \mathrm{M}$ and $4 \mu \mathrm{M}$, respectively. 
Table 1. Quantum Yield of Alexa660 or Cy5 (QY), Förster distance between Alexa660 (or Cy5) and Cy7Q $\left(\mathrm{R}_{0}\right)$, labeling efficiency of Cy7Q $(\gamma)$, excitation yield of $p \mathrm{pR}(\Psi)$, FRET efficiency $(\mathrm{E})$, change of FRET efficiency $(\Delta \mathrm{E})$, FRET-derived distance $(\mathrm{R})$, change of the distance $\left(\Delta \mathrm{R}_{\mathrm{f}}\right)$ and change of distance in crystal $\left(\Delta \mathrm{R}_{\mathrm{c}}\right)$ between $\mathrm{V} 185$ in $p \mathrm{pR}$ and indicated residues in $p \mathrm{HtrII}$

\begin{tabular}{|c|c|c|c|c|c|c|c|}
\hline$p \mathrm{HtrII}$ & \multicolumn{2}{|c|}{ V78 } & \multicolumn{2}{|c|}{ A79 } & A79 $(\text { Cy5 })^{\mathrm{c}}$ & \multicolumn{2}{|c|}{ D102 } \\
\hline QY & \multicolumn{2}{|c|}{0.24} & \multicolumn{2}{|c|}{0.32} & 0.29 & \multicolumn{2}{|c|}{0.34} \\
\hline $\mathrm{R}_{0}(\AA)$ & \multicolumn{2}{|c|}{60} & \multicolumn{2}{|c|}{65} & 64 & \multicolumn{2}{|c|}{64} \\
\hline$\gamma$ & 0.86 & 0.84 & 0.84 & 0.68 & 0.84 & 0.84 & 0.81 \\
\hline$\Psi$ & 0.52 & 0.52 & 0.52 & 0.35 & ND & 0.52 & 0.52 \\
\hline $\mathrm{E}$ & 0.55 & 0.57 & 0.62 & 0.72 & 0.73 & 0.51 & 0.57 \\
\hline$\Delta \mathrm{E}$ & -0.0012 & -0.0031 & -0.0016 & -0.0070 & ND & -0.0026 & -0.0050 \\
\hline $\mathrm{R}(\AA)^{\mathrm{a}}$ & 57 & 57 & 60 & 56 & 54 & 64 & 61 \\
\hline$\Delta \mathrm{R}_{\mathrm{f}}(\AA)^{\mathrm{a}}$ & 0.10 & 0.24 & 0.13 & 0.92 & ND & 0.23 & 0.42 \\
\hline$\Delta \mathrm{R}_{\mathrm{c}}(\AA)^{\mathrm{b}}$ & \multicolumn{2}{|c|}{0.27} & \multicolumn{3}{|c|}{0.54} & \multicolumn{2}{|c|}{ ND } \\
\hline
\end{tabular}

Alexa660 and Cy7Q were used as a donor and acceptor, respectively.

a) The distances were calculated under the assumption of isotropic orientation of dyes, i.e. $\kappa^{2}=2 / 3$ (see methods).

b) Data represent the distance changes measured from the two crystal structures in the ground state and the M state (3).

c) The value was obtained with Cy5/Cy7Q pair. 


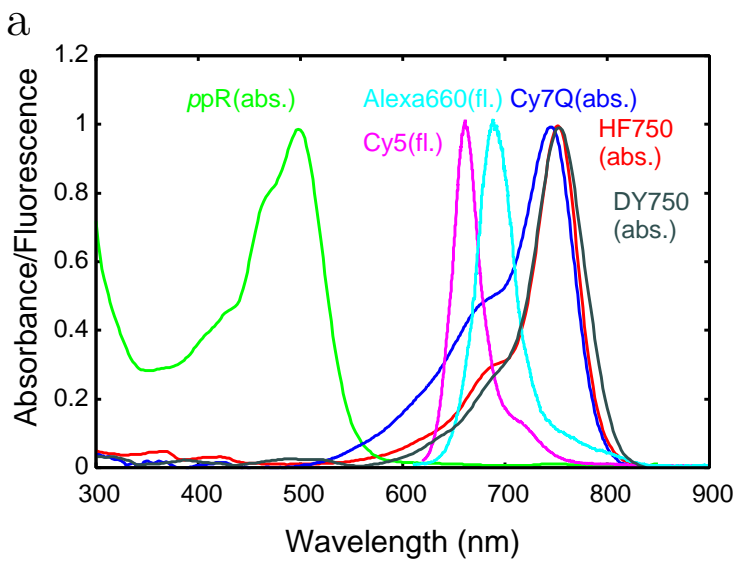

b
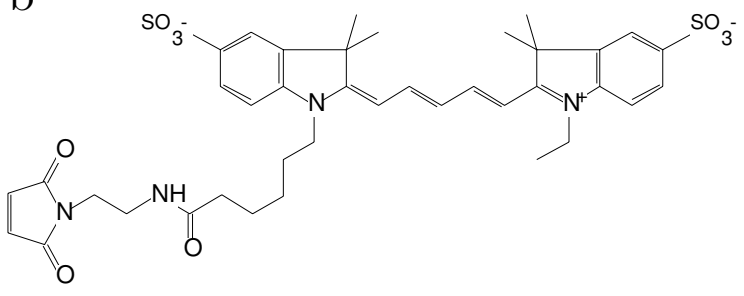

C

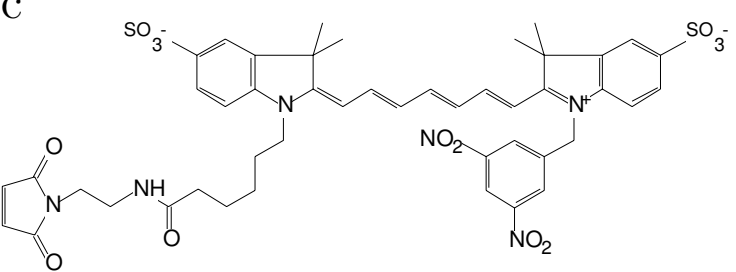

d

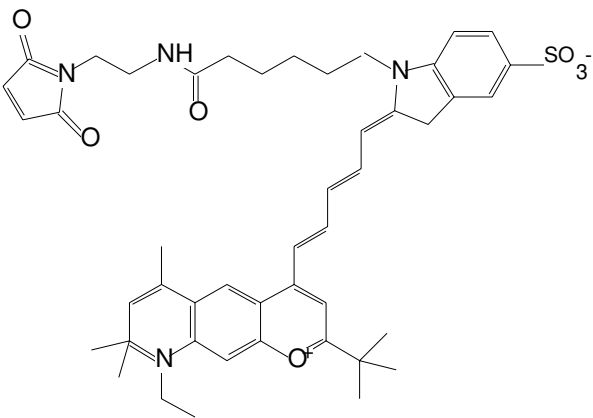

Fig. 1 


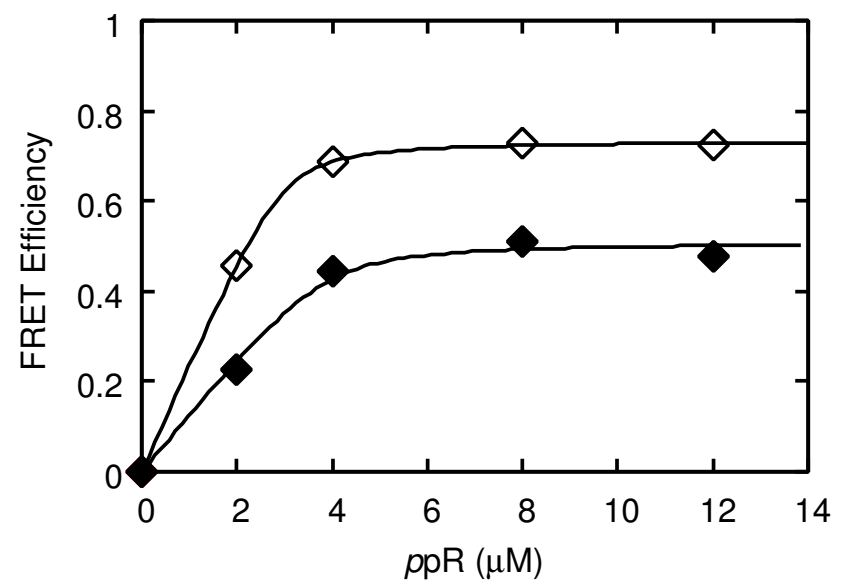

Fig. 3 
a

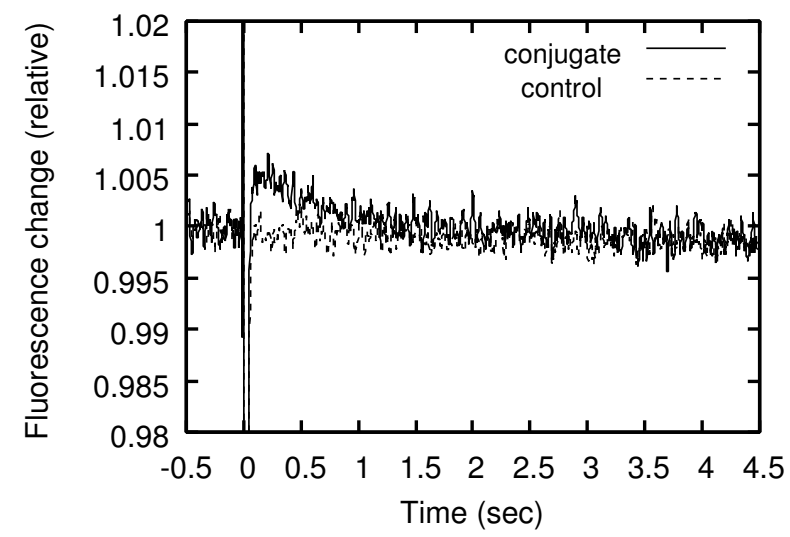

b

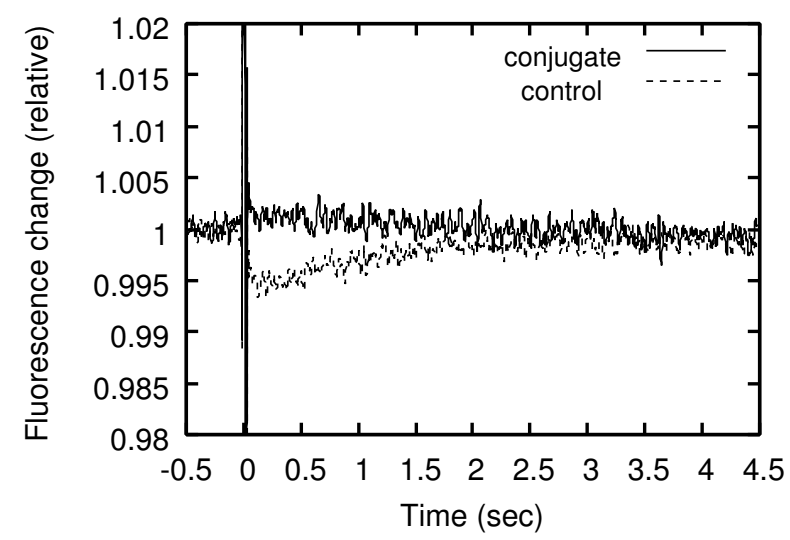

c

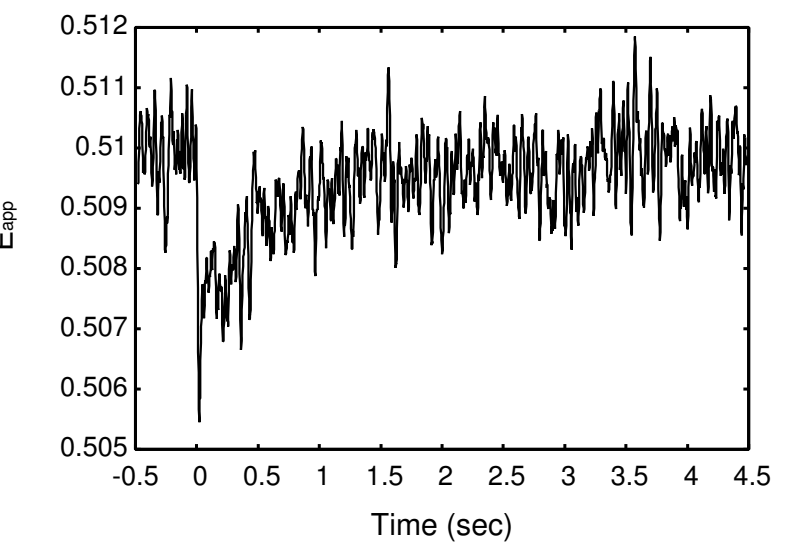

d

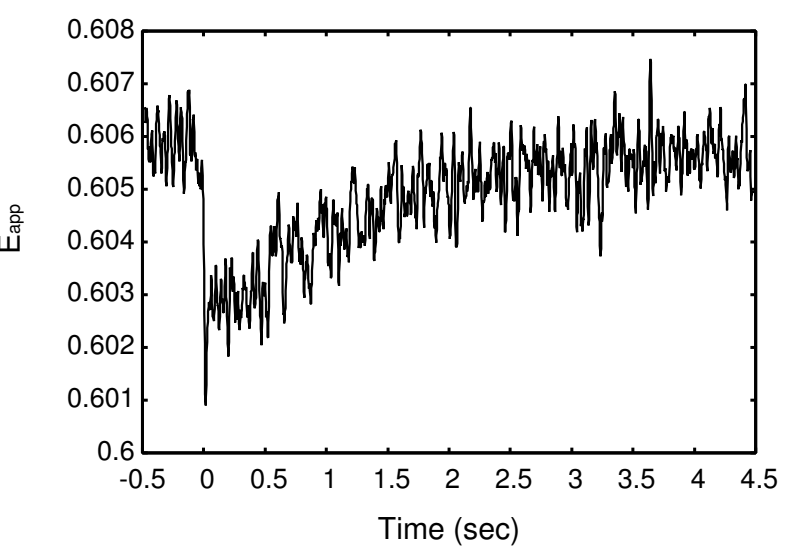

Fig. 4 